\title{
PLAN DE ESTUDIOS BASADO EN COMPETENCIAS \\ PARA LA CARRERA DE NUTRICIÓN Y DIETÉTICA DE LA UNIVERSIDAD DE CONCEPCIÓN
}

\section{COMPETENCY-BASED CURRICULUM FOR UNDERGRADUATE PROGRAM IN NUTRITION AND DIETETICS OF THE UNIVERSIDAD OF CONCEPCION}

\author{
Ana María Labraña T. (1), Eliana Durán F. (1), \\ Gisela Asenjo I. (1), Gunhild Hansel R. (2)
}

\begin{abstract}
(1) Departamento de Bromatología, Nutrición y Dietética, Facultad de Farmacia, Universidad de Concepción, Concepción, Chile.

(2) Dirección de Docencia, Universidad de Concepción, Concepción, Chile.
\end{abstract}

\begin{abstract}
The objective of this paper was to describe the process of developing a competency-based curriculum for the Undergraduate Program in Nutrition and Dietetics of the University of Concepción. To obtain the information required to structure the new curriculum, the "Developing a Curriculum" methodology was used. Semi-structure interviews and expert panel were used to collect information that was then used to develop the profile of a Nutritionist in Chile and a graduate profile. Subsequently, a new competency-based 5-year curriculum was proposed based on the identified profiles. The quantitative results were analyzed and interpreted with the statistical software SPSS Version 10.0 and the qualitative analysis was classification of answers according to category. The competencybased curriculum, the profile of nutritionist, and the graduate profile were implemented in 2007.
\end{abstract}

Keywords: Professional profile, graduate profile, curriculum, competencies.

Este trabajo fue recibido el 9 de Junio de 2009 y aceptado para ser publicado el 2 de Julio de 2010.

\section{INTRODUCCIÓN}

El modelo educativo de la Universidad de Concepción surge como respuesta a las demandas del contexto cultural, de desarrollo, ocupacionales y de la masificación educacional de los últimos años, orientadas hacia una educación centrada en el aprendizaje $(1,2)$. Esto involucra entre otros, cambios en los procesos formativos, en la concepción curricular basada en competencias y en resultados de aprendizajes, en nuevas formas de aprender y de evaluar como así mismo implica un modelo educativo pluridimensional y la vinculación de las actividades académicas, laborales, de investigación y de valores, en el proceso de formación profesional. En este escenario la carrera de Nutrición y Dietética de la Facultad de Farmacia, cuyo objetivo es formar Nutricionistas con sólidas competencias profesionales, que insertos en el sistema alimentario nutricional, sea capaz de actuar en las áreas de demanda, consumo y utilización biológica de los alimentos, para promover un óptimo estado nutricional y de salud, en la población (3), ha desarrollado un proyecto conducente a la innovación curricular que permita cumplir con el modelo educativo (4).

De esta forma la carrera de Nutrición y Dietética pretende constituirse en la opción preferencial, para muchos estudiantes que provienen de la enseñanza media y que aspiran a estudiar en ella, para transformarse en profesionales con estudios marcados por los conceptos de excelencia, innovación, calidad, flexibilidad, movilidad estudiantil, sistema de créditos transferibles y con posibilidades de articulación con el post grado (1). 
Por lo anterior, este trabajo tuvo como objetivo elaborar un Plan de Estudios basado en competencias para la carrera de Nutrición y Dietética de la Universidad de Concepción, que dé cuenta de los nuevos desafíos que enfrenta la educación superior en Chile.

\section{MATERIALES Y MÉTODOS}

Para iniciar el proceso de elaboración de Plan de Estudios basado en competencias a nivel institucional, se realizó un taller de sensibilización con docentes y autoridades, con el objeto de dar a conocer los nuevos lineamientos y políticas de la casa de estudios que dicen relación con la formulación de currículos basados en competencias $(5,6)$. Como primer paso se procedió a la socialización y capacitación de los docentes adscritos a la carrera en las nuevas metodologías para proceder a iniciar el proceso de generación del nuevo Plan de Estudios.

Para la obtención del perfil del nutricionista, también llamado perfil profesional, se trabajó con la metodología Developing A Curriculum( DACUM )(7), que consiste en analizar un puesto de trabajo en los distintos ámbitos laborales, en este caso, delineados en tres áreas: nutrición clínica, nutrición en salud pública y en alimentación colectiva. La validación del perfil profesional se realizó con un panel de 8 expertos externos en la disciplina, y se definieron las funciones y tareas específicas, las áreas de interés y de desarrollo del nutricionista. Una vez validado el perfil, se procedió al diseño de un instrumento para investigar la demanda laboral de las competencias requeridas en el ámbito nacional y regional en el campo de la nutrición y dietética. Se aplicó una encuesta, por técnica de entrevista semiestructurada, a una muestra de 58 personas pertenecientes a las 3 áreas principales de desempeño, distribuidos en 42 nutricionistas y 16 empleadores. Con los resultados de la investigación en terreno se estructuró el perfil profesional y luego el perfil de egreso. Con este último se procedió a diseñar el plan de estudios basado en competencias, el cual requirió de la realización de 50 talleres con una asesora experta en la formulación de currículo por competencias, en los que participaron todos los docentes adscritos a la carrera. En

\section{TABLA 1}

\section{Perfil profesional del nutricionista en Chile.}

\section{COMPETENCIAS}

1. Evaluar el estado nutricional individual y grupal

2. Proporcionar atención dietética y dieto terapéutica al ser humano a través del ciclo vital.

3. Diagnosticar la situación alimentario nutricional de la comunidad

4. Educar al paciente y su familia para lograr cambios de conducta alimentaria, individual o grupal.

5. Realizar visitas domiciliarias integrales.

6. Capacidad de auto perfeccionarse permanentemente.

7. Participar en los programas nacionales de promoción de salud y prevención de enfermedades

8. Gestionar de proceso de producción en servicios de alimentación.

9. Administrar fondos y gestionar recursos humanos.

10. Capacidad de comunicarse e interactuar profesionalmente con pacientes y su entorno familiar, clientes, a nivel institucional, intersectorial, intercultural e interdisciplinario.

11. Trabajar en equipo interdisciplinarios y en redes

12. Trabajar en contextos interculturales

13. Formular nuevos productos alimenticios

14. Elaborar y gestionar proyectos en el área de la nutrición y la dietética.

15. Investigar en el área de la alimentación, nutrición y la dietética.

16. Capacitar agentes multiplicadores y personal técnico en temas de alimentación y nutrición.

17. Manejo del marco legal y normas y estándares, en el ámbito de la nutrición y dietética en Chile.

18. Manejo del inglés técnico y de la computación

19. Aplicar la ética profesional en el ejercicio de la profesión. 
estos, se elaboraron los programas de cada asignatura y los respectivos módulos, según el formato establecido por la Dirección de Docencia de la Universidad de Concepción (5).

El análisis e interpretación de los resultados se realizó con software estadístico SPSS versión 10.0 para la distribución de frecuencias, porcentajes y otras mediciones cuantitativas. El análisis cualitativo se realizó por clasificación de las respuestas por categorías.

\section{RESULTADOS Y DISCUSIÓN}

De los resultados del DACUM y de la investigación en el campo laboral se obtuvieron los dos perfiles para diseñar el plan de estudios basado en competencias, destacando en su elaboración el alto interés, y el fuerte compromiso personal e institucional de los actores involucrados. La tabla 1 muestra el perfil profesional del Nutricionista en Chile, el que quedó constituido por 19 competencias, tanto genéricas como específicas, que le permitirán cumplir su rol profesional en forma competente y ajustada a los requerimientos del mercado laboral y al actual perfil epidemiológico y nutricional que posee hoy el país.

En la tabla 2, se detalla el perfil de egreso de la carrera de Nutrición y Dietética de la Universidad de Concepción, compuesto por 15 competencias específicas

\section{TABLA 2}

\section{Perfil del egresado de la Carrera de Nutrición y Dietética de la Universidad de Concepción.}

\section{COMPETENCIAS ESPECIFICAS}

1. Brindar atención dietética y dieto terapéutica a través de todo el ciclo vital con enfoque de salud holístico y con especial atención a la diversidad.

2. Determinar la situación alimentaria nutricional de la comunidad acotada a partir de los indicadores correspondientes.

3. Evaluar el estado nutricional en personas sanas o enfermos en diversos contextos.

4. Elaborar y aplicar microproyectos en el área de la nutrición y la dietética.

5. Capacitar agentes multiplicadores y personal técnico en el área de la nutrición y la dietética.

6. Utilizar elementos administrativos básicos en programas alimentarios y de salud.

7. Participar en la asistencia nutricional intensiva durante todo el ciclo vital.

8. Realizar visitas domiciliarias integrales con enfoque de salud holístico.

9. Capacidad básica de investigar en el área de la nutrición y la dietética: estudio de casos, familia y comunidad

10. Capacidad de supervisar los procesos de calidad en la producción y distribución de los alimentos.

11. Utilizar técnicas de planificación de minutas alimentarias.

12. Calcular el aporte nutricional de los alimentos y preparaciones culinarias.

13. Capacidad de gestionar la prescripción dietética para estructurar el apoyo nutricional.

14. Participar en el proceso de producción en servicios de alimentación colectiva.

15. Manejo de normas de normas y estándares en el ámbito de la nutrición y la dietética.

\section{COMPETENCIAS GENERALES}

16. Capacidad de aprender y actualizarse permanentemente

17. Compromiso ético en el desempeño de sus diversas actividades profesionales

18. Capacidad de comunicarse en forma efectiva en el área de la nutrición y la dietética.

19. Capacidad de integrar y trabajar en equipo .en el área de la nutrición y la dietética.

20. Desarrollar habilidades interpersonales para el trabajo autónomo y proactivo.

21. Capacidad para resolver problemas inherentes a la disciplina.

22. Manejo del inglés técnico y de las técnicas de la comunicación y la informática. 
y 7 competencias generales, entregando en estas últimas las herramientas para lograr un trabajo en equipo, donde la ética y la comunicación aparecen como primordiales en todo el quehacer profesional. Las competencias especificas están orientadas al cumplimento de la esencia en la formación profesional en este caso de un Nutricionista (8-12) y a los actuales requerimientos del mercado laboral para este profesional, teniendo en consideración que se trata de un recién egresado.

En la tabla 3, se muestra el plan de estudios, con un diseño basado en competencias, con una duración de 5 años, y la respectiva clasificación establecida por la Dirección de Docencia de la Universidad de Concepción, $(2,5,6,13)$. Se observa que los módulos y asignaturas se organizan por años de estudios y por tipos de competencias a lograr, se contemplan actividades prácticas a partir desde el primer año de estudios, las que son progresivas y de carácter integral ya que le permitirán al estudiante aplicar los conocimientos, habilidades y actitudes adquiridas hasta el momento. Además incluye diferentes tipos de módulos; fundamentales, de especia- lidades, multidisciplinario, de apoyo, y de habilidades transferibles. Los módulos están articulados por años de estudios y por eje temático, este último establecido para cada año de formación y en congruencia con los resultados de aprendizajes y las competencias a lograr.

El plan de estudios resultante obedece a un modelo educativo centrado en el estudiante $(1,2)$, apunta a cubrir los nuevos requerimientos de la disciplina y se priorizan aprendizajes significativos. La propuesta curricular presenta un desarrollo equitativo en 3 ámbitos de acción: nutrición clínica, nutrición salud pública, y alimentación colectiva.

La nueva estructura curricular es más flexible, abierta y ajustada a requerimientos del mercado laboral y al perfil epidemiológico y nutricional del país, permitiendo una formación holística, tal como sucede en países del hemisferio norte en el que estas estructuras curriculares han sido probadas $(8,10,14)$. Por otra parte este nuevo paradigma educativo permitirá a su vez, la adquisición de competencias de constante actualización, para que el titulado brinde una atención profesional de alto nivel.

TABLA 3

Plan de estudios basado en competencias. Nutrición y Dietética U de C 2007.

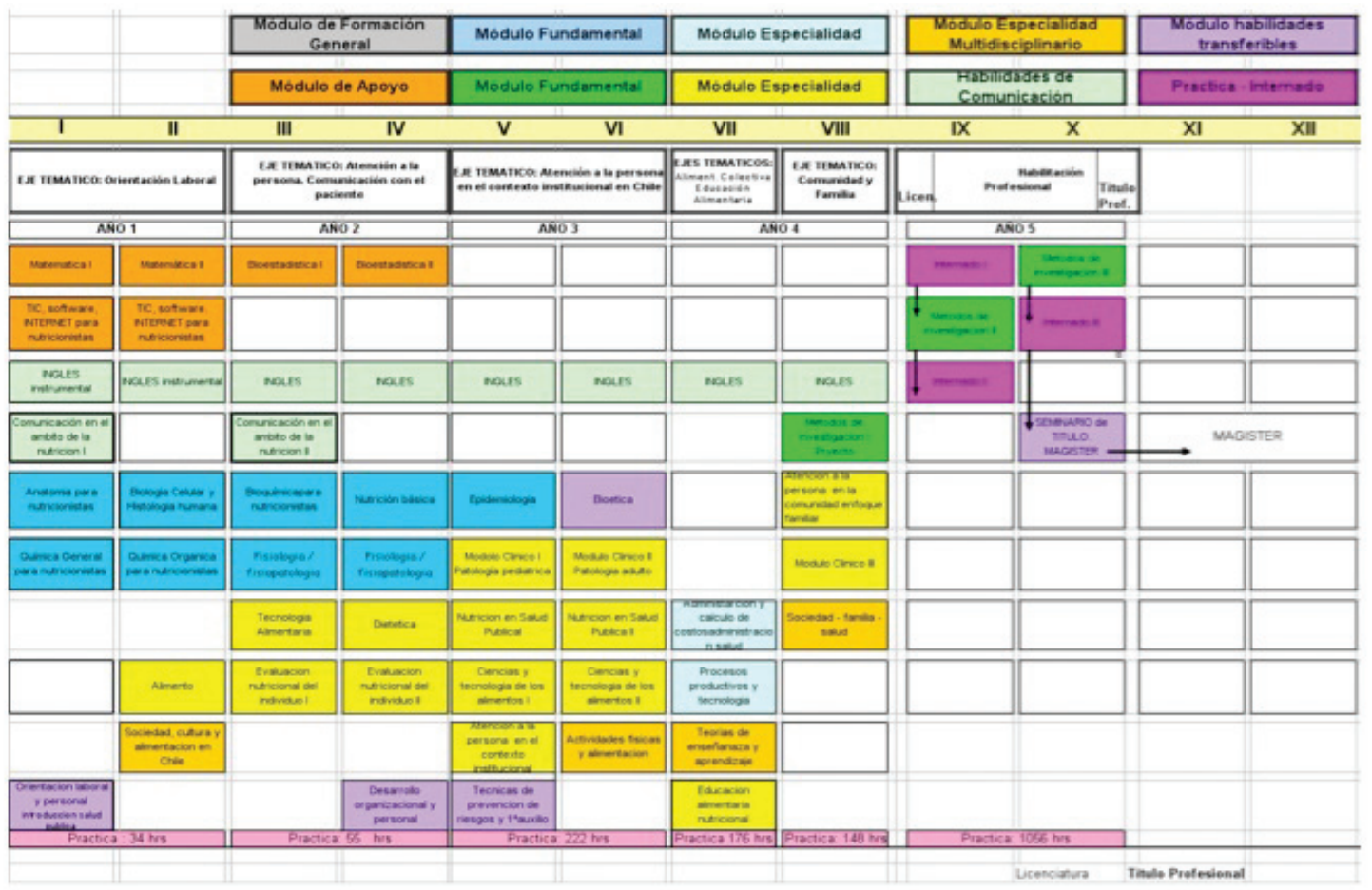




\section{CONCLUSIÓN}

Las universidades enfrentan hoy un cambio de época, caracterizado por una gran complejidad, incertidumbre, impredecibilidad, turbulencias y caos. El surgimiento de la sociedad del conocimiento, la globalización, el impacto del conocimiento científico, tecnológico y la internacionalización, se enmarcan en el modelo de mercado, donde la búsqueda de calidad es exigencia de todo el sistema social y por ende del educacional (1). Por lo anterior es inminente procurar excelencia en la formación académica, para satisfacer las exigencias del mercado laboral y la competitividad en la cual el ser humano esta inserto. El nuevo currículo aquí presentado, permite a los Nutricionistas recién egresados, obtener las competencias requeridas para enfrentar los actuales escenarios y vertiginosos cambios, conservando con mayor profundidad el sello institucional de la Universidad de Concepción en concordancia a su modelo educativo y a la misión y visión de la misma $(15,16)$. Se concluye que la carrera de Nutrición y Dietética de la Universidad de Concepción, cuenta con una propuesta curricular basada en competencias, desde el año 2007, la que estará en constante revisión y ajuste para responder a los nuevos escenarios y requerimientos del mercado laboral y de la educación superior en Chile, expresada en este caso en un proyecto Mecesup en red de innovación curricular actualmente en curso (17).

\section{RESUMEN}

El presente trabajo tuvo como objetivo elaborar un plan de estudios basado en competencias para la carrera de Nutrición y Dietética de la Universidad de Concepción. Para la obtención de la información básica necesaria para la estructuración del nuevo currículo, se utilizó la metodología Developing A Curriculum. Se realizaron entrevistas semiestructuradas y se desarrolló un panel de expertos, para la obtención del perfil del nutricionista en Chile y el perfil de egreso. Con estos, se elaboró la nueva propuesta curricular basada en competencias con una duración de 5 años. El análisis e interpretación de los resultados cuantitativos se realizó con software estadístico SPSS versión 10.0 y el análisis cualitativo se realizó por clasificación de las respuestas por categorías. Se concluye que la carrera de Nutrición y Dietética, de la Universidad de Concepción, cuenta con un perfil de nutricionista, perfil de egreso y un plan de estudios basado en competencias, desde el año 2007.

Palabras clave: Perfil profesional, perfil de egreso, plan de estudios, competencias.

Dirigir la correspondencia a:

Profesora
Ana María Labraña Torres.

Depto. Bromatología, Nutrición y Dietética

Facultad de Farmacia

Universidad de Concepción

Casilla 237, Concepción.

Fono: 041- 2206510

Fax: 041- 2210516.

E-mail: alabrana@udec.cl

Agradecimientos: Las autoras agradecen la valiosa colaboración y participación de todos los profesores adscritos a la carrera de Nutrición y Dietética de la Universidad de Concepción, a los colaboradores docentes, a los nutricionistas de servicios y empresas y a los empleadores.

\section{BIBLIOGRAFÍA}

1. Dirección de Docencia, Universidad de Concepción, Boletín de la Dirección de Docencia, julio 2008.

2. Centro Interuniversitario de Desarrollo-CINDA. Grupo Operativo de Universidades Chilenas. Fondo de Desarrollo Institucional-Mineduc-Chile. Diseño Curricular basado en Competencias y Aseguramiento de la calidad en la educación Superior, pág 291. Santiago, Marzo de 2009.

3. Torres, M S. Plan de Estudios 2004, Carrera de Nutrición y Dietética, Facultad de Farmacia, Universidad de Concepción.

4. Labraña, AM. Actualización de la malla curricular de la Carrera de Nutrición y Dietética de la Universidad de Concepción, según las exigencias de la reforma educacional chilena y el proyecto Tuning. Proyecto de Docencia tipo B, No 06-83, Dirección de Docencia, UdeC, 2007.

5. Dirección de Docencia, Universidad de Concepción, Reglamento de Docencia de Pregrado, Decreto U. de C. N1 ${ }^{\circ}$ 2008-116, 2009.

6. Dirección de Docencia, Universidad de Concepción, Boletín de la Dirección de Docencia, Marzo 2009.

7. Mertens, L. DACUM y sus variantes SCID y AMO. CINTERFOR-OIT. November 1997.

8. Díaz, E y cols. Colegio de Nutricionistas de Chile A. G. El nutricionista aspectos específicos de la disciplina. Chile, Santiago de Chile, 2007.

9. Labraña A., Durán E., Soto D. Competencias del nutricionista en el ámbito de atención primaria de salud. Rev Chil Nutr. 2005; 32 (3), 239-46.

10. Crovetto M y cols. Perfil ocupacional por competencias del nutricionista. Taller metodológico, Colegio de Nutricionistas de Chile. Santiago- Chile. Mayo 2006.

11. Soto D, Olivares S. Diagnóstico de la situación del 
Nutricionista en Chile. Rev OPS Bol. 1982; 12:452454.

12. Olivares, S. Soto D, Zacarías I Valoración del nutricionista: formación y campos de acción. Cuadernos Médicos Sociales, 1993 pp. 87-94.

13. OPS/OMS. Manual metodológico para el estudio de los perfiles ocupacional, profesional y académico del Nutricionista- Dietista en los países de América Latina. Bogotá: Oficina Sanitaria Panamericana, 1990.

14. González, M., Cifuentes, M. Una experiencia en la formación por competencias en nutrición y Dietética en Canadá y Estados Unidos. CINDA, Santiago,
Marzo 2009.

15. Universidad de Concepción, Plan Estratégico Institucional 2006-2010.

16. Proyecto Tuning. Informe Final Proyecto Tuning América Latina 2004-2007. Reflexiones y Perspectivas de la Educación Superior. España, Universidad de Deusto, 2007.

17. Proyecto Mecesup en Red UBB0606 Innovación académica para la optimización de la formación de nutricionistas. Universidad del Bio- Bio, Universidad de Valparaíso, Universidad de Concepción. 2007-2010. 